\title{
Lithium treatment in bipolar adolescents: a follow-up naturalistic study
}

This article was published in the following Dove Press journal:

Neuropsychiatric Disease and Treatment

\author{
Gabriele Masi' \\ Annarita Milone' \\ Giulia Scrinzi ${ }^{2}$ \\ Maria Mucci' \\ Valentina Viglione' \\ Gabriella Bruni' \\ Stefano Berloffa' \\ Simone Pisano 3 \\ 'IRCCS Stella Maris Foundation, \\ Developmental Psychiatry and \\ Psychopharmacology Unit, Pisa, \\ Italy; ${ }^{2}$ Child Neuropsychiatry Unit, \\ Department of Surgical Sciences, \\ Dentistry, Gynecology and Pediatrics, \\ University of Verona, Verona, Italy; \\ ${ }^{3}$ Clinic of Child and Adolescent \\ Neuropsychiatry, Department of \\ Medicine and Surgery, University of \\ Salerno, Salerno, Italy
}

Background: Although lithium is currently approved for the treatment of bipolar disorders in youth, long term data, are still scant. The aim of this study was to describe the safety and efficacy of lithium in referred bipolar adolescents, who were followed up at the 4th (T1) and 8th (T2) month of treatment.

Methods: The design was naturalistic and retrospective, based on a clinical database, including 30 patients (18 males, mean age $14.2 \pm 2.1$ years).

Results: Mean blood level of lithium was $0.69 \pm 0.20 \mathrm{mEq} / \mathrm{L}$ at T1 and $0.70 \pm 0.18 \mathrm{mEq} / \mathrm{L}$ at T2. Both Clinical Global Impression-Severity (CGI-S) and Children Global Assessment Scale (C-GAS) scores improved from baseline (CGI-S 5.7 \pm 0.5 , C-GAS 35.1 \pm 3.7 ) to T1 (CGI-S 4.2 \pm 0.70 , C-GAS $46.4 \pm 6.5 ; P<0.001$ ), without significant differences from T1 to T2. Thyroid-stimulating hormone significantly increased from $2.16 \pm 1.8 \mathrm{mU} / \mathrm{mL}$ at baseline to $3.9 \pm 2.7 \mathrm{mU} / \mathrm{mL}$ at $\mathrm{T} 2$, remaining within the normal range, without changes in T3/T4 levels; two patients needed a thyroid hormone supplementation. Creatinine blood level did not change. No cardiac symptoms and electrocardiogram QTc changes occurred. White blood cell count significantly increased from $6.93 \pm 1.6810^{3} / \mathrm{mmc}$ at baseline to $7.94 \pm 1.9410^{3} / \mathrm{mmc}$ at $\mathrm{T} 2$, and serum calcium significantly increased from $9.68 \pm 0.3 \mathrm{mg} / \mathrm{dL}$ at baseline to $9.97 \pm 0.29 \mathrm{mg} / \mathrm{dL}$ at T2, both remaining within the normal range; all the other electrolyte levels were stable and normal during the follow-up. The treatment with lithium was well tolerated, probably due to the relatively low lithium blood levels. Gastrointestinal symptoms (16.7\%), sedation $(9.7 \%)$ and tremor $(6.4 \%)$ were the most frequently reported side effects.

Conclusion: Lithium was effective and safe in adolescent bipolar patients followed-up for eight months.

Keywords: bipolar disorder, adolescents, lithium, safety

\section{Introduction}

In the context of a multimodal approach, including psychosocial, family and psychotherapeutic interventions, the primary treatment for bipolar disorder (BD) with onset during childhood or adolescence is pharmacological. ${ }^{1}$ First-line medications include mood stabilizers, such as lithium, valproate and carbamazepine, and atypical antipsychotics, such as aripiprazole, olanzapine, quetiapine and risperidone. ${ }^{2}$ Although risperidone showed higher response rates in bipolar adolescents, compared to both lithium and divalproex, ${ }^{3}$ growing concerns are associated with the use of atypical antipsychotics in children and adolescents. ${ }^{4}$ Lithium is currently approved by most regulatory agencies, including the Food and Drug Administration and the European Medicine Agency, for the treatment of BD adolescents older than 12 years. Although a recent double-blind, placebo-controlled study further supported the efficacy of lithium in youths with $\mathrm{BD},{ }^{5}$ studies reporting the efficacy of lithium in youths, namely the long-term data, are still scant. ${ }^{6}$

Among the long-term studies, Strober et $\mathrm{al}^{7}$ reported on 37 adolescents with BD stabilized with lithium, who were naturalistically followed up for 18 months. At the
Correspondence: Gabriele Masi IRCCS Stella Maris Foundation, Viale del Tirreno 33I 56025 Calambrone,

Pisa, Italy

Tel +3950886111

Fax +395088630I

Email gabriele.masi@fsm.unipi.it 
end of the follow-up period, 21 patients (56.8\%) relapsed, and the relapse rate was three times higher among patients who discontinued lithium earlier. Findling et $\mathrm{al}^{8}$ explored the comparative effectiveness of lithium and divalproex in the maintenance treatment of juvenile BD, over 76 weeks, without differences in time to mood relapse or treatment discontinuation. Our aim was to describe the medium-term safety and effectiveness of lithium in referred adolescent bipolar patients, who were naturalistically followed up for 8 months.

\section{Methods}

\section{Sample and measures}

This was a naturalistic study based on a clinical database of consecutive Caucasian youth, aged between 12 and 18 years, with three inclusion criteria: a Diagnostic and Statistical Manual of Mental Disorder, Fourth Edition-Text Revised (DSM IV-TR) and DSM-5 diagnosis of BD, a pharmacological treatment with lithium carbonate, three follow-up points, at the baseline, after 4 months and 8 months. These time points were selected according to the routine follow-up procedures of our department. All the subjects were screened for psychiatric disorders, using historical information, a structured clinical interview according to DSM criteria, the Schedule for Affective Disorders and Schizophrenia for School-Age Children - Present and Lifetime Version (K-SADS-PL). ${ }^{9}$ Patients with schizophrenia, autism spectrum disorder and intellectual disability were excluded. Given the naturalistic design of the study, patients receiving other pharmacological treatments were not excluded, when the dosages of other treatments were stable from at least 4 weeks before starting lithium and remained unchanged during the follow-up. Among 45 patients who started lithium, 15 patients were excluded because their clinical condition needed the stable introduction of a new medication during the follow-up. Thirty patients remained on this treatment for at least 8 months and were included in the study (18 males, mean age of $14.2 \pm 2.1$ years). Seventeen patients $(56.7 \%)$ presented with anxiety comorbidities, 14 (46.7\%) had oppositional defiant disorder or conduct disorder and $12(40 \%)$ had an attention-deficit hyperactivity disorder (ADHD). Seventeen patients received second generation antipsychotics (SGAs), four valproic acid, two fluoxetine and 16 concomitant psychotherapy. Although our assessment was limited to the 4th and 8th month of treatment, patients monitored their blood levels of lithium more frequently, and the available data supported the good compliance with treatment.

Our primary outcome measure was the improvement in symptoms according to the Clinical Global Impression-
Severity (CGI-S) score, ${ }^{10}$ assessed at the baseline (T0), after 4 months (T1) and after 8 months (T2). Secondary outcome measure was the functional improvement assessed using the Children Global Assessment Scale (C-GAS), ${ }^{11}$ assessed at the baseline, T1 and T2. Safety data were assessed at the baseline and follow-up and included physical examination, blood cell count, blood chemistry, electrolytes, thyroid function and electrocardiogram (ECG) (including QTc interval).

All the diagnostic and therapeutic procedures, including lithium treatment, as well as the follow-up visits and data collection, were part of our routine procedures. The institutional review board of the Scientific Institute Stella Maris (Pisa) approved the study. All subjects and parents received detailed information on the assessment measures and different treatment options and gave their written informed consent to the treatment with lithium.

\section{Statistical analyses}

A paired $t$-test was used to compare the values of continuous variables from baseline to T1 and T2. Analyses were run in SPSS Version 20 (IBM Corporation, Armonk, NY, USA).

\section{Results}

Patients presented a severe baseline status (CGI-S $5.7 \pm 0.5$, C-GAS 35.1 \pm 3.7 ). Mean lithium dosage at T1 was $843 \pm 176.6 \mathrm{mg} /$ day (range 600-1,200 mg/day) and at T2 was $858 \pm 149 \mathrm{mg} /$ day (range $600-1,200 \mathrm{mg} /$ day). Mean blood level of lithium was $0.69 \pm 0.20 \mathrm{mEq} / \mathrm{L}$ at T1 and $0.70 \pm 0.18 \mathrm{mEq} / \mathrm{L}$ at T2. A summary of baseline characteristics of the sample and efficacy results is provided in Table 1.

Regarding efficacy, CGI-S score improved from 5.7 \pm 0.53 at baseline to $4.2 \pm 0.70$ at $\mathrm{T} 1(t=8.7, P<0.001)$ and $4.0 \pm 0.9$ at T2 ( $t=7.8, P<0.001$; moderately to markedly ill); from $\mathrm{T} 1$ to $\mathrm{T} 2$, the difference was not significant $(t=1.6, P=0.1)$. Similarly, C-GAS score improved from $35.1 \pm 3.7$ at baseline to $46.4 \pm 6.5$ at $\mathrm{T} 1(t=-9.0, P=0.001)$ and $47.5 \pm 8.3(t=-7.4$, $P<0.001$ ) at T2 (moderate impairment in most areas or severe in one area); from $\mathrm{T} 1$ to $\mathrm{T} 2$, the difference was not significant ( $t=-0.8 ; P=0.4$ ). No patients relapsed during the follow-up. Emergence of both suicidal ideation and behavior was not reported during the entire follow-up. The presence of any group of comorbidities (anxiety disorder, disruptive behavioral disorder and ADHD) had no effect on the results.

Regarding thyroid function, thyroid-stimulating hormone (TSH) significantly increased from $2.16 \pm 1.8 \mathrm{mU} / \mathrm{mL}$ at baseline to $3.9 \pm 2.7$ at $\mathrm{T} 1(t=-2.8, P=0.013)$ and $3.49 \pm 2.2$ at T2 $(t=-2.8 ; P=0.011)$, remaining within the normal range. 


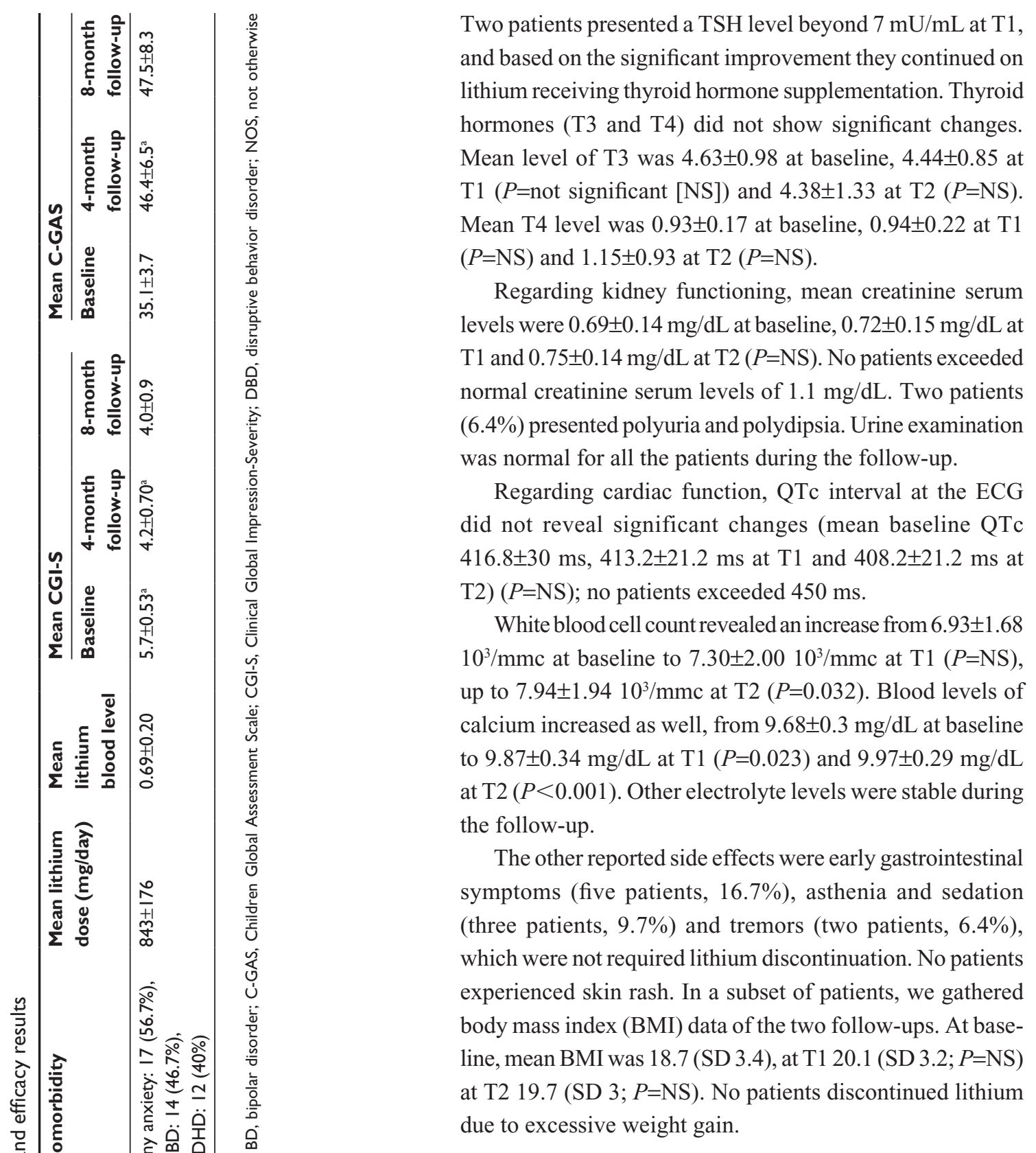

\section{Discussion}

In our sample of severely impaired adolescents with BD, lithium treatment significantly improved clinical severity and functional impairment. The clinical and functional improvement occurred in the first 4 months and was confirmed in the following 4 months, without relapses. Consistently with the antisuicidal properties of lithium in mood disorders, ${ }^{12}$ emergence of both suicidal ideation and behavior was not reported during the follow-up. It is noteworthy that effective lithium blood levels were relatively low (ranging from $0.4 \mathrm{mEq} / \mathrm{L}$ to $0.8 \mathrm{mEq} / \mathrm{L}$ ). Regarding safety data, treatment with lithium was well tolerated, which is in line with previous studies. ${ }^{6}$ A moderate increase in TSH was found, although two patients 
needed the add-on thyroid hormone supplementation. Renal function was preserved. Neither cardiac symptoms nor QTc alterations emerged during the follow-up. White blood cell count and calcium levels increased, but within the normal range and without clinical implications. Other electrolyte levels (including sodium and potassium) remained normal during the follow-up. Gastrointestinal symptoms usually occurred in the early phase of treatment, while sedation and tremor were more rarely reported during the follow-up. The rate of side effects was lower, compared to similar studies in adult populations, probably due to relatively lower blood levels of lithium in our sample of adolescents.

Our naturalistic study presents several methodological limitations. This is not a randomized, controlled study. We only gathered data from patients who completed 8 months of follow-up, and this may have biased the results in favor of better efficacy and safety. Another confusing element is that many patients received other medications, namely SGAs or valproic acid, with anti-manic properties even in monotherapy. However, the dosage of other medications was stable from at least 4 weeks before starting lithium and remained unchanged during the follow-up. Although not supported by all guidelines, polypharmacy is the rule rather than exception in the real world of bipolar patients receiving medications. In the Bhangoo et al's ${ }^{13}$ study, youths were receiving $3.40 \pm 1.48$ medications, and $77 \%$ of them had had a trial of an antipsychotic. In the Pavuluri et al's s ${ }^{14}$ study, only $17.5 \%$ of bipolar youths without psychotic symptoms were effectively controlled by a monotherapy with a mood stabilizer for at least 6 months, while $66.3 \%$ of those receiving a combination of a mood stabilizer and an SGA were responders. Another relevant limitation, considering the high rate of comorbidities, is that we have used the CGI-S, CGI-I and C-GAS as outcome measures, which are not specific for manic/mixed symptoms' severity and improvement, but give an overall rating of effectiveness. An improvement in the global measure scores may have been affected by other comorbid disorders and not by a specific effect of lithium on BD. However, the CGI and C-GAS criterion corresponds to what clinicians use to determine whether to continue or interrupt a medication trial in naturalistic settings, as the course of the clinical picture as a whole is more reliably captured by a more global measure. Finally, we cannot extend our findings to longer follow-ups, as patients were subsequently monitored and cared by their own territorial facilities. In our study, all the patients were treated as needed (mono- or polypharmacy) and followed up in a routine clinical setting, and this may actually be one of the strengths of our study. Long-term naturalistic perspective studies might represent an important source of information on effectiveness and safety of treatment under ordinary clinical conditions, and the present findings are therefore of particular relevance to clinical practice in treating child and adolescent BD. In addition, the reassuring safety profile allows to speculate early intervention with lithium especially in special populations with juvenile bipolar patients, including patients who suffered from traumatic experiences, ${ }^{15}$ or those with high suicidal risk, ${ }^{12}$ or with comorbid conduct disorder and substance abuse. ${ }^{16}$

\section{Acknowledgments}

We would like to thank the European Research Project MATRICS (Multidisciplinary Approaches to Translational Research in Conduct Syndromes) Work Package 6-1European Community (grant agreement no: 603016) for the support in the publication of the study. This study did not receive any specific grant from funding agencies in the public, commercial or not-for-profit sectors.

\section{Disclosure}

Dr GM was in the advisory boards for Eli Lilly, Shire and Angelini, has received research grants from Eli Lilly, Shire and Lundbeck and has been speaker for Eli Lilly, Shire and FB Health. The other authors report no conflicts of interest in this work.

\section{References}

1. McClellan J, Kowatch R, Findling RL, Work Group on Quality Issues. Practice parameter for the assessment and treatment of children and adolescents with bipolar disorder. J Am Acad Child Adolesc Psychiatry. 2007;46(1):107-125.

2. Liu HY, Potter MP, Woodworth KY, et al. Pharmacologic treatments for pediatric bipolar disorder: a review and meta-analysis. J Am Acad Child Adolesc Psychiatry. 2011;50(8):749-762.

3. Geller B, Luby JL, Joshi P, et al. A randomized controlled trial of risperidone, lithium, or divalproex sodium for initial treatment of bipolar I disorder, manic or mixed phase, in children and adolescents. Arch Gen Psychiatry. 2012;69(5):515-528.

4. Fraguas D, Correll CU, Merchán-Naranjo J, et al. Efficacy and safety of second-generation antipsychotics in children and adolescents with psychotic and bipolar spectrum disorders: comprehensive review of prospective head-to-head and placebo-controlled comparisons. Eur Neuropsychopharmacol. 2011;21(8):621-645.

5. Findling RL, Robb A, Mcnamara NK, et al. Lithium in the acute treatment of bipolar I disorder: A double-blind, placebo-controlled study. Pediatrics. 2015;136(5):885-894.

6. Pisano S, Pozzi M, Catone G, et al. Putative mechanisms of action and clinical use of lithium in children and adolescents: a critical review. Curr Neuropharmacol. Epub 2017 Dec 19.

7. Strober M, Morrell W, Lampert C, Burroughs J. Relapse following discontinuation of lithium maintenance therapy in adolescents with bipolar I illness: a naturalistic study. Am J Psychiatry. 1990;147(4):457-461.

8. Findling RL, Mcnamara NK, Youngstrom EA, et al. Double-blind 18-month trial of lithium versus divalproex maintenance treatment in pediatric bipolar disorder. J Am Acad Child Adolesc Psychiatry. 2005; 44(5):409-417. 
9. Kaufman J, Birmaher B, Brent D, et al. Schedule for Affective Disorders and Schizophrenia for School-Age Children-Present and Lifetime Version (K-SADS-PL): initial reliability and validity data. J Am Acad Child Adolesc Psychiatry. 1997;36(7):980-988.

10. Guy W. ECDEU Assessment Manual for Psychopharmacology, Revised. Rockville, MD: US Department of Health, Education and Welfare; 1976.

11. Shaffer D, Gould MS, Brasic J, et al. A children's global assessment scale CGAS. Arch Gen Psychiatry. 1983;40(11):1228-1231.

12. Cipriani A, Hawton K, Stockton S, Geddes JR. Lithium in the prevention of suicide in mood disorders: updated systematic review and meta-analysis. BMJ. 2013;346:f3646.

13. Bhangoo RK, Lowe CH, Myers FS, et al. Medication use in children and adolescents treated in the community for bipolar disorder. J Child Adolesc Psychopharmacol. 2003;13(4):515-522.
14. Pavuluri MN, Henry DB, Carbray JA, et al. A one-year open-label trial of risperidone augmentation in lithium nonresponder youth with preschool-onset bipolar disorder. J Child Adolesc Psychopharmacol. 2006;16(3):336-350.

15. Janiri D, Sani G, Rossi P, et al. Amygdala and hippocampus volumes are differently affected by childhood trauma in patients with bipolar disorders and healthy controls. Bipolar Disord. 2017;19(5):353-362.

16. Geller B, Cooper TB, Sun K, et al. Double-blind and placebo-controlled study of lithium for adolescent bipolar disorders with secondary substance dependency. J Am Acad Child Adolesc Psychiatry. 1998; 37(2):171-178.
Neuropsychiatric Disease and Treatment

\section{Publish your work in this journal}

Neuropsychiatric Disease and Treatment is an international, peerreviewed journal of clinical therapeutics and pharmacology focusing on concise rapid reporting of clinical or pre-clinical studies on a range of neuropsychiatric and neurological disorders. This journal is indexed on PubMed Central, the 'PsycINFO' database and CAS,

\section{Dovepress}

and is the official journal of The International Neuropsychiatric Association (INA). The manuscript management system is completely online and includes a very quick and fair peer-review system, which is all easy to use. Visit http://www.dovepress.com/testimonials.php to read real quotes from published authors.

Submit your manuscript here: http://www.dovepress.com/neuropsychiatric-disease-and-treatment-journal 